\title{
Understanding Deep Brain Stimulation: In Vivo Metabolic Consequences of the Electrode Insertional Effect
}

\author{
Marta Casquero-Veiga $\left(D,{ }^{1}\right.$ David García-García, ${ }^{1,2,3}$ \\ Manuel Desco $(\mathbb{D})^{1,2,3,4}$ and María Luisa Soto-Montenegro $\mathbb{D}^{1,2}$ \\ ${ }^{1}$ Instituto de Investigación Sanitaria Gregorio Marañón, Madrid 28007, Spain \\ ${ }^{2}$ CIBER de Salud Mental (CIBERSAM), Madrid 28029, Spain \\ ${ }^{3}$ Departamento de Bioingeniería e Ingeniería Aeroespacial, Universidad Carlos III de Madrid, Leganés 28911, Spain \\ ${ }^{4}$ Centro Nacional de Investigaciones Cardiovasculares (CNIC), Madrid 28029, Spain \\ Correspondence should be addressed to Manuel Desco; desco@hggm.es
}

Received 26 April 2018; Revised 10 September 2018; Accepted 1 October 2018; Published 17 October 2018

Academic Editor: Diane Ruge

Copyright (C) 2018 Marta Casquero-Veiga et al. This is an open access article distributed under the Creative Commons Attribution License, which permits unrestricted use, distribution, and reproduction in any medium, provided the original work is properly cited.

\begin{abstract}
Deep brain stimulation (DBS) is a neurosurgery technique widely used in movement disorders, although its mechanism of action remains unclear. In fact, apart from the stimulation itself, the mechanical insertion of the electrode may play a crucial role. Here we aimed to distinguish between the insertional and the DBS effects on brain glucose metabolism. To this end, electrodes were implanted targeting the medial prefrontal cortex in five adult male Wistar rats. Positron Emission Tomography (PET) studies were performed before surgery (D0) and seven (D7) and nine days (D9) after that. DBS was applied during the ${ }^{18}$ FDG uptake of the D9 study. PET data were analysed with statistical parametric mapping. We found an electrode insertional effect in cortical areas, while DBS resulted in a more widespread metabolic pattern. The consequences of simultaneous electrode and DBS factors revealed a combination of both effects. Therefore, the insertion metabolic effects differed from the stimulation ones, which should be considered when assessing DBS protocols.
\end{abstract}

\section{Introduction}

In recent years, brain stimulation techniques have emerged in bioscientific and clinical scenarios. Deep brain stimulation (DBS) is a technique that modulates neuronal discharge patterns through electrical current both locally, at the electrode implantation site, and also in remote brain areas associated with the deep brain target $[1,2]$. The success and safety offered by DBS in movement disorders [3] have led to consider its potential application in other neurological and mental pathologies, such as psychiatric disorders [4-6], with the subsequent search for new DBS targets. However, the mechanism of action of DBS remains unclear and depends on two confounded factors: the electrode insertion per se and the electrical stimulation. Indeed, certain symptomatology improvement has been related to the mere insertion of the electrodes in the treatment of epilepsy [7] and chronic neuropathic pain in humans [8]. Also, antidepressant-like effects have been found in rats in which electrodes were implanted, but without applying electrical stimulation [9]. To our knowledge, these are the only studies that have shown this insertional effect, but none of them has studied the subsequent brain regional activity modulation. Thus, the aim of this study is to assess the insertional effect of the electrode, isolated from the acute electrical stimulation itself, on brain glucose metabolism studied by positron emission tomography (PET) and statistical parametric mapping (SPM) techniques in rats with electrodes placed in the medial prefrontal cortex (mPFC).

\section{Materials and Methods}

2.1. Animals. Adult male Wistar rats $(\sim 350 \mathrm{~g})(\mathrm{N}=5)$ were housed in a temperature- and humidity-controlled vivarium, 


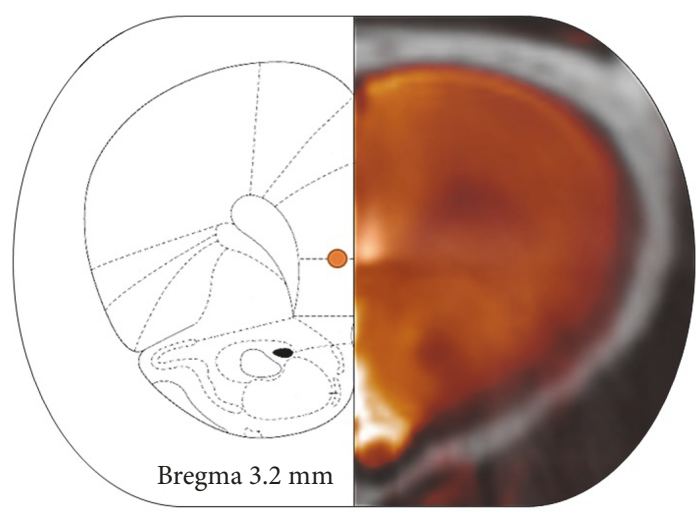

FIGURE 1: Electrode placement. Axial view of a CT scan registered to the MR template of an animal (right) and its correspondent slice in the Paxinos \& Watson atlas [10] (left), to verify the correct electrode location in the $\mathrm{mPFC}$. The bright and orange points represent the electrode tip in the $\mathrm{CT}$ and atlas images, respectively.

on a $12 \mathrm{~h}$ light-dark cycle, with standard laboratory rat chow and water ad libitum. Animals were deprived of food 68 hours prior to the PET study. All experimental animal procedures were conducted according to the European Communities Council Directive 2010/63/EU and approved by the Ethics Committee for Animal Experimentation of the Hospital Gregorio Marañón.

This study was performed following the guidelines established by the principles of the 3Rs to minimize the number of animals included in this work [11]. Nevertheless, considering the longitudinal design of this research, the number of animals was sufficient to obtain enough statistically significant differences between time points.

2.2. Stereotaxic Surgery and DBS Protocol. Animals were anesthetized with ketamine/xylazine $(100 / 10 \mathrm{mg} / \mathrm{kg})$. Concentric bipolar platinum-iridium electrodes (Plastics One, Roanoke, USA) were bilaterally implanted targeting the mPFC $(+3.5 \mathrm{~mm}$ posterior, $+0.6 \mathrm{~mm}$ lateral from Bregma, $3.4 \mathrm{~mm}$ ventral from Dura) [10]. Electrodes were fixed to the skull surface with dental acrylic cement (Technovit ${ }^{\circledR}$, Germany). Antibiotic (ceftriaxone, $100 \mathrm{mg} / \mathrm{kg}$ ) and analgesic (buprenorphine, $0.1 \mathrm{mg} / \mathrm{kg}$ ) drugs were administered for 3 days as postoperative care.

DBS was applied during the radiotracer uptake period (45 $\mathrm{min}$ ) with an isolated stimulator (CS 120 8i, CIBERTEC S.A., Spain) in a constant current mode at $130 \mathrm{~Hz}, 150 \mu \mathrm{A}$ and a pulse width of $100 \mu \mathrm{s}$.

2.3. Imaging Studies. PET and computerized tomography (CT) scans were acquired just before surgery (D0, baseline) and 7 days (D7, without stimulation) and 9 days (D9, with stimulation) after that, in order to provide enough time for surgical recovery [12]. Additional CT scans were acquired at the end of the surgery to verify the correct placement of the electrodes (Figure 1). In addition, one magnetic resonance imaging (MRI) scan of a single nonoperated animal was acquired to be used as an anatomical template.
Animals were scanned using a small-animal PET/CT scanner (ARGUS PET/CT, SEDECAL, Madrid), under anaesthesia with isoflurane (3\% induction, $1.5 \%$ maintenance in $100 \%$ O2). 2-Deoxy-2- $\left[{ }^{18} \mathrm{~F}\right]$ fluoro-D-glucose (FDG, $37 \mathrm{Mbq}$ ) was intravenously injected and, after $45 \mathrm{~min}$ of uptake, animals were scanned for $40 \mathrm{~min}$. Images were reconstructed using a 2D-OSEM algorithm, with a spatial resolution of $1.45 \mathrm{~mm}$ Full Width Half Maximum (FWHM), a voxel size of $0.3875 \times 0.3875 \times 0.775 \mathrm{~mm}^{3}$, and an energy window of 400-700 keV. Decay and dead-time corrections were applied.

CT studies were acquired with the same scanner, using the following parameters: $340 \mathrm{~mA}, 40 \mathrm{kV}, 360$ projections, 8 shots, and pixel size of $200 \mu \mathrm{m}$. Images were reconstructed using an FDK algorithm (isotropic voxel size of $0.124 \mathrm{~mm}$ ) [13].

The MRI study was acquired with a 7-Tesla Biospec 70/20 scanner (Bruker, Ettlingen, Germany). A T2-weighted spinecho sequence was acquired with $\mathrm{TE}=33 \mathrm{~ms}, \mathrm{TR}=3732 \mathrm{~ms}$, and a slice thickness of $0.8 \mathrm{~mm}$ ( 34 slices). The matrix size was $256 \times 256$ pixels with a FOV of $3.5 \times 3.5 \mathrm{~cm} 2$.

2.4. Analysis of PET Data. PET images postprocessing and voxel value normalization were performed following the protocols previously described by our group [14, 15]. Briefly, PET images were spatially coregistered to a random reference CT scan (CTref) and smoothed with an isotropic Gaussian kernel of $2 \mathrm{~mm}$ FWHM. A brain mask segmented in the MRI, also registered to the CTref was applied to all PETs to exclude voxels outside the brain. Voxel values were normalized to average intensity of a brain region without statistically significant differences between groups [15].

The statistical analysis consisted on a voxel-wise analysis of PET data using SPM12 (http://www.fil.ion.ucl.ac.uk/spm/ software/spm12/) by means of paired T-tests, setting a significance threshold of $\mathrm{p}<0.005$ uncorrected (voxel-level significance), but cluster-based corrected in order to avoid type II errors [16]. Only clusters higher than 50 adjacent voxels were considered aiming at reducing type I error.

We performed three different comparisons to evaluate the modulatory effect of the electrode insertion (D0 versus D7, study I), the stimulation (D7 versus D9, study II), and the combination of both the insertion and the stimulation (D0 versus D9, study III), on brain metabolism.

In this sense, we assume that the metabolic differences we show in the study II are almost completely due to the acute effect of the high-frequency electrical stimulation. Although the microlesional effect related to the electrode insertion is highly variable between subjects [8], the stimulation effect has been shown to be much stronger that the insertional one [17], and this latter tends to reduce over time. Furthermore, both the insertion (D7) and the stimulation (D9) PET acquisitions were separated by just two days, period in which no new relevant consequences derived from the electrode presence are expected. In this context, although a late effect of the insertion could have appeared, its influence on the study II would be minimum and possibly masked by the impact of a stronger stimulus represented by the application of highfrequency electrical stimulation. 

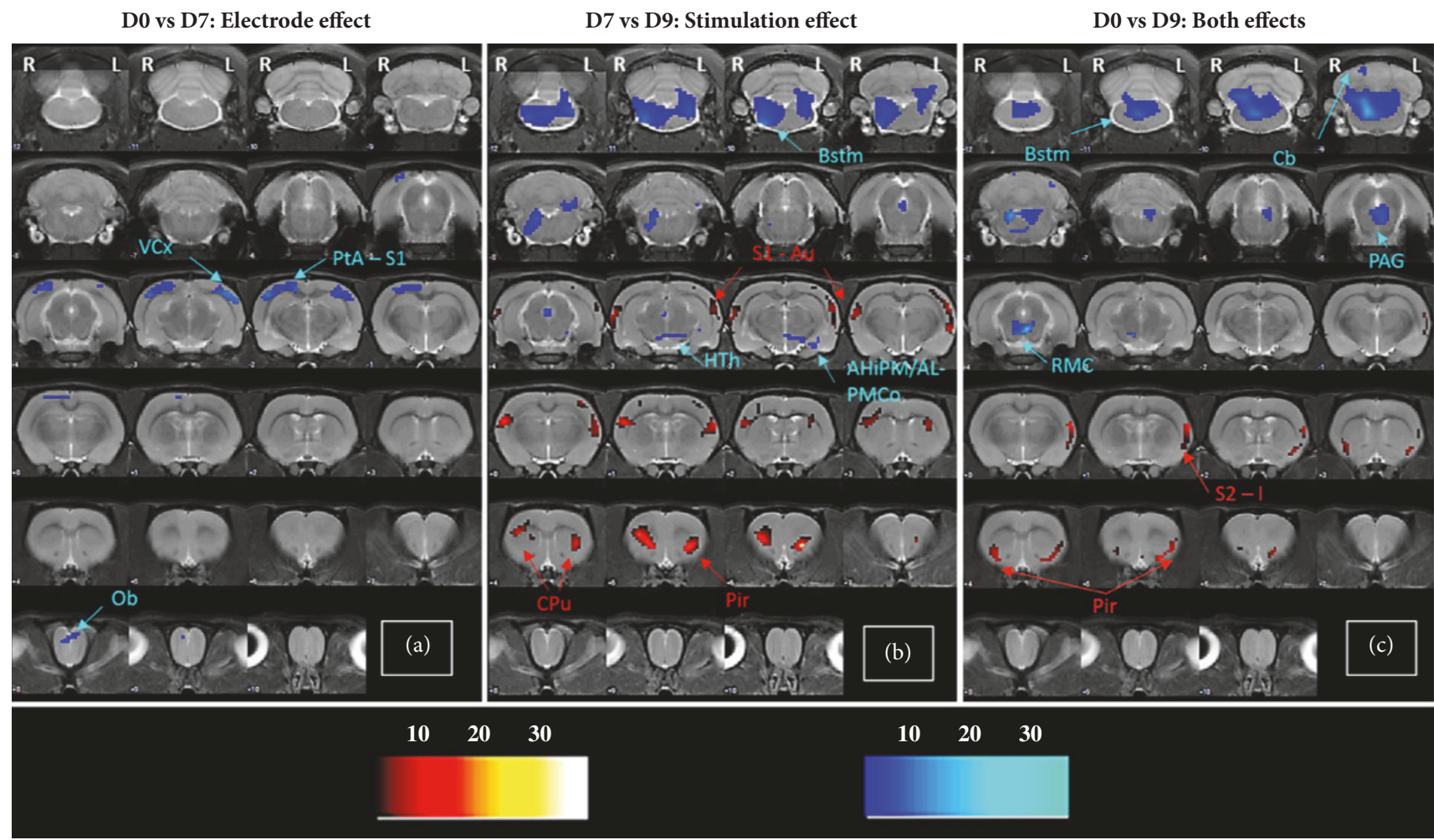

$\begin{array}{lll}10 & 20 & 30\end{array}$

FIGURE 2: Changes in brain metabolic activity. Voxel based SPM results in T-maps overlaid on a T2 MR image, showing the changes in glucose metabolism due to electrodes insertion (a), stimulation (b), and both effects (c). The color bars in the right represent the $\mathrm{T}$ values corresponding to lower (blue) and higher (red) FDG uptake ( $\mathrm{p}$-value $<0.005$ (unc.); $\mathrm{k}=50$ voxels). Glucose metabolism: increase (hot colors); decrease (cold colors) [AHiPM/AL: amydalohippocampal area posteromedial/anterolateral part, Au: auditory cortex, Bstm: brainstem, Cb: cerebellum, CPu: caudate-putamen, HTh: hypothalamus, I: insular cortex, Ob: olfactory bulb, PAG: periaqueductal grey matter, Pir: piriform cortex, PMCo: posteromedial cortical amygdaloid nucleus, PtA: parietal association cortex, RMC: red nucleus, S1: primary somatosensory cortex, S2: secondary somatosensory cortex, and VCx: visual cortex].

\section{Results}

3.1. Electrode Insertion Effect. The presence of the electrodes (D0 versus D7, study I) led to a reduced FDG uptake in parietal association (PtA), primary somatosensory (S1), and visual cortices (Figure 2(a); Table 1(A)).

3.2. Stimulation Effect. The electrical stimulation (D7 versus D9, study II) led to a decreased FDG uptake in brainstem (Bstm), amygdaloid nuclei (AHiAL, AHiPM, and PMCo), and hypothalamus (HTh), together with an increased metabolism in caudate-putamen $(\mathrm{CPu})$, piriform (Pir), S1, and auditory cortex (Au) (Figure 2(b); Table 1(B)).

3.3. Insertion and Stimulation Effect. The combination of both effects (D0 versus D9, study III) showed a decreased FDG uptake in Bstm, red nucleus (RMC), and periaqueductal grey matter (PAG) and higher metabolism in secondary somatosensory (S2), insular (I), and Pir cortices (Figure 2(c), Table $1(\mathrm{C})$ ).

\section{Discussion}

First, we describe an insertional effect on brain glucose metabolism in sensory areas that are connected to mPFC [18].
Second, mPFC-DBS resulted in a distinct brain metabolic pattern, with more brain areas affected than in study I. Thus, DBS induced changes in circuits where the mPFC plays a key role, such as limbic ( $\mathrm{AHi}, \mathrm{PMCo}$, and $\mathrm{Pir}$ ) and reward $(\mathrm{CPu}$ and Bstm) systems [18]. Finally, the simultaneous consequences of the electrodes and the stimulation revealed lower cortical activation compared to the study II, showing a compensation of the hypometabolism derived from the electrodes presence (study I). Specifically, the absence of metabolic changes in S1 shown in study III exemplifies this mechanism, as this structure showed a metabolic reduction and an increase in studies I and II, respectively. Moreover, S1 is the only region in which there is an overlap between both effects.

The insertion effect could appear in response to the microlesion induced by the electrode in the mPFC [7] and the subsequent inflammation of the targeted area [9]. Thus, although the microlesion effect fades away over time [19], the clinical manifestations of the insertional effect could persist from days to months (exceptionally, years), or even being absent, despite comparing patients under the same surgical protocol and disease [8]. Besides, other authors have also provided evidence of its permanence on the healthy rat brain metabolism beyond one week after surgery [12]; although, in contrast to our findings, they showed similar effects of stimulation and insertion, being the latter of lower intensity. 
TABLE 1: Changes in brain metabolism due to electrode (A), stimulation (B), and both effects (C).

\begin{tabular}{|c|c|c|c|c|c|c|c|}
\hline ROI & Side & $\mathrm{T}$ & $\mathbf{k}$ & $\downarrow / \uparrow$ & $\begin{array}{c}\mathbf{p}_{\text {unc }} \text { peak } \\
\text { level }\end{array}$ & $\begin{array}{c}\text { FWE } \\
\text { peak level }\end{array}$ & $\begin{array}{c}\text { FWE } \\
\text { cluster level }\end{array}$ \\
\hline \multicolumn{8}{|c|}{ (A) D0 vs D7: Electrode effect } \\
\hline $\mathrm{Ob}$ & $\mathrm{R} \& \mathrm{~L}$ & 15.68 & 121 & $\downarrow$ & $<0.001$ & 0.811 & 0.067 \\
\hline PtA - S1 & $\mathrm{R}$ & 14.97 & 365 & $\downarrow$ & $<0.001$ & 0.880 & $<0.001$ \\
\hline $\mathrm{VCx}$ & $\mathrm{L}$ & 14.75 & 184 & $\downarrow$ & $<0.001$ & 0.884 & 0.015 \\
\hline \multicolumn{8}{|c|}{ (B) D7 vs D9: Stimulation effect } \\
\hline Bstm & $\mathrm{R} \& \mathrm{~L}$ & 18.39 & \multirow{2}{*}{1549} & $\downarrow$ & $<0.001$ & 0.432 & \multirow[b]{2}{*}{$<0.001$} \\
\hline $\begin{array}{l}\text { AHiPM/AL- } \\
\text { PMCo - } \\
\text { HTh }\end{array}$ & $\mathrm{L}$ & 10.39 & & $\downarrow$ & $<0.001$ & 0.949 & \\
\hline $\mathrm{CPu}$ & \multirow{2}{*}{$\mathrm{L}$} & 37.56 & \multirow{2}{*}{738} & $\uparrow$ & $<0.001$ & 0.025 & \multirow{2}{*}{$<0.001$} \\
\hline $\mathrm{S} 1-\mathrm{Au}$ & & 10.53 & & $\uparrow$ & $<0.001$ & 0.947 & \\
\hline $\mathrm{CPu}-\mathrm{Pir}$ & \multirow{2}{*}{$\mathrm{R}$} & 17.74 & \multirow{2}{*}{695} & $\uparrow$ & $<0.001$ & 0.497 & \multirow{2}{*}{$<0.001$} \\
\hline $\mathrm{S1-Au}$ & & 10.45 & & $\uparrow$ & $<0.001$ & 0.948 & \\
\hline \multicolumn{8}{|c|}{ (C) D0 vs D9: Both effects } \\
\hline RMC - PAG & $\mathrm{R} \& \mathrm{~L}$ & 26.24 & \multirow{2}{*}{1430} & $\downarrow$ & $<0.001$ & 0.105 & \multirow{2}{*}{$<0.001$} \\
\hline $\mathrm{Cb}$ & $\mathrm{R}$ & 5.90 & & $\downarrow$ & 0.002 & 0.998 & \\
\hline S2 - I & $\mathrm{L}$ & 15.20 & \multirow{2}{*}{475} & $\uparrow$ & $<0.001$ & 0.892 & \multirow{2}{*}{$<0.001$} \\
\hline Pir & $\mathrm{L}$ & 9.10 & & $\uparrow$ & $<0.001$ & 0.979 & \\
\hline Pir & $\mathrm{R}$ & 12.96 & 152 & $\uparrow$ & $<0.001$ & 0.929 & 0.026 \\
\hline
\end{tabular}

Structures: AHiPM/AL: amydalohippocampal area posteromedial/anterolateral part, Au: auditory cortex, Bstm: brainstem, Cb: cerebellum, CPu: caudateputamen, HTh: hypothalamus, I: insular cortex, Ob: olfactory bulb, PAG: periaqueductal gray matter, Pir: piriform cortex, PMCo: posteromedial cortical amygdaloid nucleus, PtA: parietal association cortex, RMC: red nucleus, S1: primary somatosensory cortex, S2: secondary somatosensory cortex, and VCx: visual cortex.

ROI: region of interest. Side: right $(R)$ and left $(L)$. T: t value; k: cluster size. Glucose metabolism: increase $(\uparrow)$ and decrease $(\downarrow)$. punc: p-value uncorrected; FWE: family wise error correction.

Furthermore, comparable results have been also shown in Parkinson disease (PD) patients after electrode insertion in the subthalamic nucleus, which resulted in similar but lower metabolic changes than subthalamotomy in PD-related pattern, while no significant clinical effect was observed due to the insertion [20]. Conversely, task-fMRI data found partial differences between the insertion and stimulation consequences in PD [19].

Therefore, the wide variability showed in relation to the clinical and physiological consequences of the electrode insertion could be highly dependent on several factors (e.g., the health state of the subjects, the DBS target selected, the number of microelectrode recording trajectories performed during the surgery [20], the time elapsed between the surgery and the test, etc.). In fact, PFC input and output connections shared with the sensory cortex occupy different locations and ordering [21], which is not common to other regions and could suppose a substantial difference regarding DBS effect. Thus, the opposed metabolism caused in the somatosensory cortex by the electrode placement and the stimulation alone could respond to the recent neural informative disruption theories of DBS mechanism of action [22, 23]. Importantly, these changes would have not been uncovered without a 3-times longitudinal design. Taken all together, both stimulation and insertion results seem to involve the same brain networks, although in a considerably different extent. This information would be helpful for adjusting the DBS protocols. Thus, understanding the regions affected by each involved factor (insertion; stimulation), together with the intensity and direction (activation; inhibition) of the produced modulation, could lead to more specific and efficient DBS protocols.

\section{Limitations}

Our work is subjected to several limitations. On the one hand, the small sample size selected, which responds to the aim of providing preliminary evidences that we considered to be important for understanding the DBS mechanism of action through a metabolic perspective. Therefore, we applied strict statistical thresholds in order to show more accurate results, which lead us to discard potentially important effects that do not reach statistical significance. In fact, the electrode insertion has been proved to produce lower metabolic changes than subthalamotomy or stimulation $[12,20]$, which could be masked in the present study. These points would explain why, in contrast with previous literature, the metabolic pattern observed with the insertion differs from that caused by the stimulation.

On the other hand, we have only included healthy animals in our study, which does not allow us to extrapolate the observed changes to a disease model due to the differences 
related to a diseased brain. Nevertheless, we aimed to describe the metabolic consequences of electrode insertion and electrical stimulation, excluding any other intervening factors, in order to isolate those effects and improve their metabolic characterization.

\section{Conclusions}

In conclusion, our study highlights the importance of the design of appropriate protocols, particularly in neuroimaging, emphasizing the value of scanning the same subject with/without DBS, for a full understanding of the DBS mechanism of action and its clinical consequences. This will allow taking advantage of the electrodes and the stimulation consequences in order to optimize the DBS protocols for achieving the desired therapeutic effects.

\section{Data Availability}

The data used to support the findings of this study are included within the article.

\section{Conflicts of Interest}

The authors declare that there are no conflicts of interest regarding the publication of this paper.

\section{Acknowledgments}

The authors thank Kenia Martinez for her help in performing the statistical analyses and the interpretation of the results; Alexandra de Francisco and Yolanda Sierra for their support in stereotaxic surgery, animal handling, and acquisition of imaging studies. This research was supported by Fundación Mapfre, Alicia Koplowitz [FAK16/01], CIBER de Salud Mental (CIBERSAM), the Ministry of Economy and Competitiveness ISCIII-FIS Grants [PI14/00860, CPII14/00005, and PI17/01766], and Delegación del Gobierno para el Plan Nacional sobre Drogas [PNSD 2017/085] and cofinanced by ERDF (FEDER) Funds from the European Commission, "A way of Making Europe," and Comunidad de Madrid [BRADE-CM S2013/ICE-2958].

\section{References}

[1] J. Kuhn, W. Gaebel, J. Klosterkoetter, and C. Woopen, "Deep brain stimulation as a new therapeutic approach in therapyresistant mental disorders: Ethical aspects of investigational treatment," European Archives of Psychiatry and Clinical Neurosciences, vol. 259, no. 2, pp. S135-S141, 2009.

[2] J. Kuhn, T. O. Gründler, D. Lenartz, V. Sturm, J. Klosterkötter, and W. Huff, "Deep Brain Stimulation for Psychiatric Disorders," Deutsches Aerzteblatt Online, 2010.

[3] R. S. Shah, S. Chang, H. Min, Z. Cho, C. D. Blaha, and K. H. Lee, "Deep Brain Stimulation: Technology at the Cutting Edge," Journal of Clinical Neurology, vol. 6, no. 4, p. 167, 2010.

[4] T. Sankar, T. S. Tierney, and C. Hamani, "Novel applications of deep brain stimulation," Surgical Neurology International, vol. 3, pp. S26-S33, 2012.
[5] J. Guridi and G. Aldave, "Dianas quirúrgicas en el tratamiento de enfermedades psiquiátricas: Desde el movimiento a las emociones," Neurocirugía, vol. 22, no. 1, 2011.

[6] C. B. Mikell, S. Sinha, and S. A. Sheth, "Neurosurgery for schizophrenia: an update on pathophysiology and a novel therapeutic target," Journal of Neurosurgery, vol. 124, no. 4, pp. 917-928, 2016.

[7] V. Krishna, N. K. King, F. Sammartino et al., "Anterior Nucleus Deep Brain Stimulation for Refractory Epilepsy," Neurosurgery, vol. 78, no. 6, pp. 802-811, 2016.

[8] C. Hamani, J. M. Schwalb, A. R. Rezai, J. O. Dostrovsky, K. D. Davis, and A. M. Lozano, "Deep brain stimulation for chronic neuropathic pain: Long-term outcome and the incidence of insertional effect," PAIN, vol. 125, no. 1-2, pp. 188-196, 2006.

[9] L. Perez-Caballero, R. Pérez-Egea, C. Romero-Grimaldi et al., "Early responses to deep brain stimulation in depression are modulated by anti-inflammatory drugs," Molecular Psychiatry, vol. 19, no. 5, pp. 607-614, 2014.

[10] G. Paxinos and C. Watson, The Rat Brain in Stereotaxic Coordinates, Academic Press, 4th edition, 1998.

[11] National Centre for the Replacement Refinement \& Reduction of Animals in Research, The 3Rs, 2017, https://www.nc3rs.org.uk/ the-3rs.

[12] N. Van Den Berge, V. Keereman, C. Vanhove et al., "Hippocampal Deep Brain Stimulation Reduces Glucose Utilization in the Healthy Rat Brain," Molecular Imaging and Biology, vol. 17, no. 3, pp. 373-383, 2015.

[13] M. Abella, J. J. Vaquero, A. Sisniega et al., "Software architecture for multi-bed FDK-based reconstruction in X-ray CT scanners," Computer Methods and Programs in Biomedicine, vol. 107, no. 2, pp. 218-232, 2012.

[14] M. L. Soto-Montenegro, J. Pascau, and M. Desco, "Response to Deep Brain Stimulation in the Lateral Hypothalamic Area in a Rat Model of Obesity: In Vivo Assessment of Brain Glucose Metabolism," Molecular Imaging and Biology, pp. 830-837, 2014.

[15] J. Gasull-Camós, M. L. Soto-Montenegro, M. Casquero-Veiga, M. Desco, F. Artigas, and A. Castañé, "Differential patterns of subcortical activity evoked by glial GLT-1 blockade in prelimbic and infralimbic cortex: Relationship to antidepressant-like effects in rats," The International Journal of Neuropsychopharmacology, vol. 20, no. 12, pp. 988-993, 2017.

[16] A. Verger, C. Stegmayr, N. Galldiks et al., "Evaluation of factors influencing 18F-FET uptake in the brain," NeuroImage: Clinical, vol. 17, pp. 491-497, 2018.

[17] T. Tykocki, P. Nauman, H. Koziara, and T. Mandat, "Microlesion effect as a predictor of the effectiveness of subthalamic deep brain stimulation for Parkinson's disease," Stereotactic and Functional Neurosurgery, vol. 91, no. 1, pp. 12-17, 2013.

[18] J. C. Klein, M. F. S. Rushworth, T. E. J. Behrens et al., “Topography of connections between human prefrontal cortex and mediodorsal thalamus studied with diffusion tractography," NeuroImage, vol. 51, no. 2, pp. 555-564, 2010.

[19] R. Jech, K. Mueller, D. Urgošík et al., "The Subthalamic Microlesion Story in Parkinson's Disease: Electrode InsertionRelated Motor Improvement with Relative Cortico-Subcortical Hypoactivation in fMRI," PLoS ONE, vol. 7, no. 11, 2012.

[20] M. Pourfar, C. Tang, T. Lin, V. Dhawan, M. G. Kaplitt, and D. D. Eidelberg, "Assessing the microlesion effect of subthalamic deep brain stimulation surgery with FDG PET,' Journal of Neurosurgery, vol. 110, no. 6, pp. 1278-1282, 2009.

[21] S. A. Bedwell, E. E. Billett, J. J. Crofts, and C. J. Tinsley, "The topology of connections between rat prefrontal, motor and sensory cortices," Frontiers in Systems Neuroscience, vol. 8, 2014. 
[22] C. C. McIntyre and R. W. Anderson, "Deep brain stimulation mechanisms: the control of network activity via neurochemistry modulation," Journal of Neurochemistry, pp. 338-345, 2016.

[23] S. Chiken and A. Nambu, "Mechanism of Deep Brain Stimulation: Inhibition, Excitation, or Disruption?" The Neuroscientist, vol. 22, no. 3, pp. 313-322, 2015. 


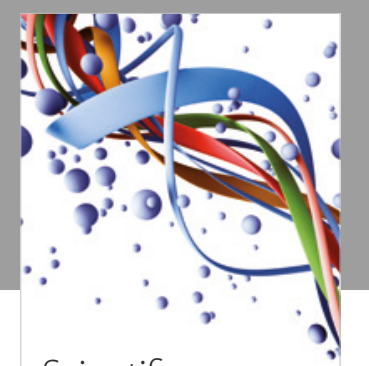

Scientifica
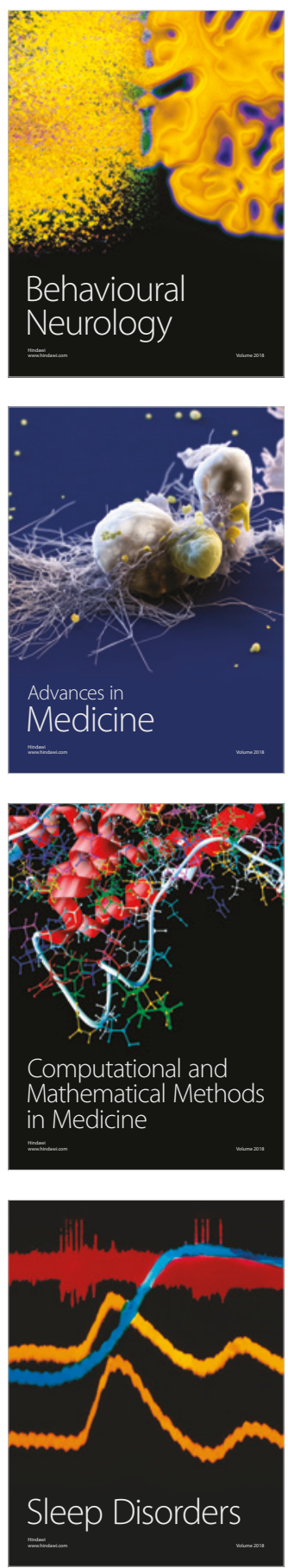

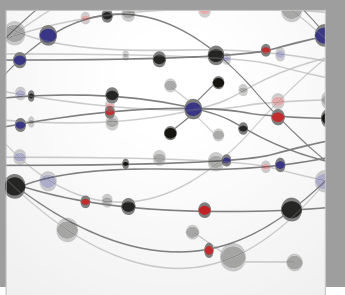

The Scientific World Journal

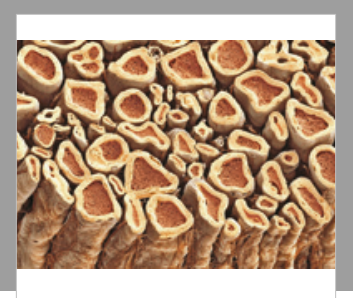

Case Reports in

Neurological Medicine

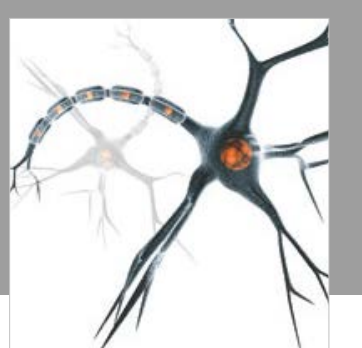

Neural Plasticity

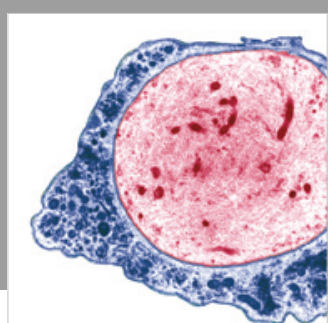

Multiple Sclerosis

International

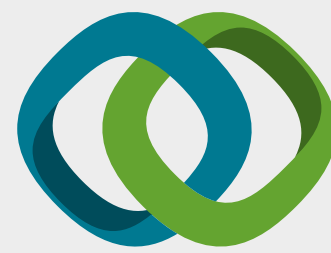

Hindawi

Submit your manuscripts at

www.hindawi.com
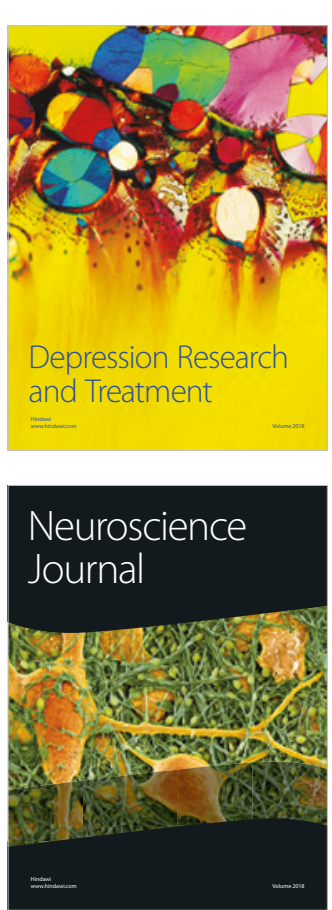

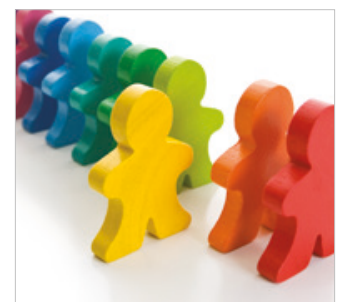

Autism

Research and Treatment
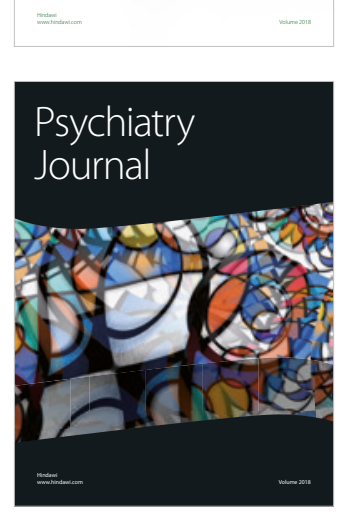
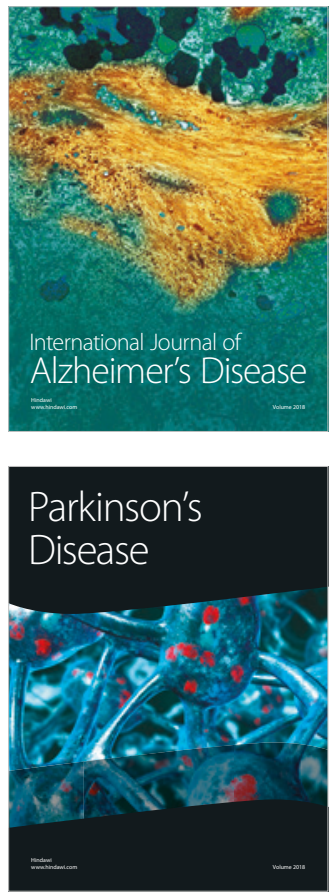
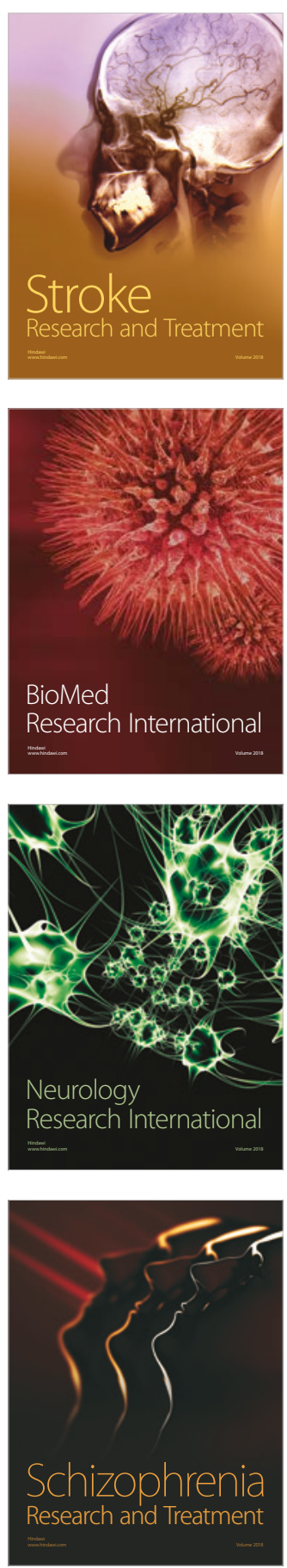